\title{
Lactobacillus rhamnosus and its cell-free culture supernatant differentially modulate inflammatory biomarkers in Escherichia coli-challenged human dendritic cells
}

\author{
Miriam Bermudez-Brito $^{1 *}$, Sergio Muñoz-Quezada ${ }^{1}$, Carolina Gomez-Llorente $^{1}$, Fernando Romero ${ }^{2}$ \\ and Angel Gil ${ }^{1}$ \\ ${ }^{1}$ Department of Biochemistry and Molecular Biology II, Institute of Nutrition and Food Technology "José Mataix" (INyTA), \\ Biomedical Research Center, University of Granada, Avenida del Conocimiento s/n, Armilla 18100, Granada, Spain \\ ${ }^{2}$ Hero Global Technology Centre For Infant Nutrition, Hero Group, Avenida del Murcia 1, Alcantarilla 30820, Murcia, Spain
}

(Submitted 4 July 2013 - Final revision received 14 November 2013 - Accepted 9 December 2013 - First published online 30 January 2014)

\section{Abstract}

The intestinal immune system maintains a delicate balance between immunogenicity against invading pathogens and tolerance to the commensal microbiota and food antigens. Different strains of probiotics possess the ability to finely regulate the activation of dendritic cells (DC), polarising the subsequent activity of T-cells. Nevertheless, information about their underlying mechanisms of action is scarce. In the present study, we investigated the immunomodulatory effects of a potentially probiotic strain, Lactobacillus rhamnosus CNCM I-4036, and its cell-free culture supernatant (CFS) on human DC challenged with Escherichia coli. The results showed that the levels of pro-inflammatory cytokines such as IL-1 $\beta$, IL-6, IL-8 and IL-12p70 were higher in the cells treated with live L. rhamnosus than in the cells treated with the CFS. In the presence of $E$. coli, the supernatant was more effective than the probiotic bacteria in reducing the secretion of pro-inflammatory cytokines. In addition, live L. rhamnosus potently induced the production of transforming growth factor (TGF)- $\beta 1$ and TGF- $\beta 2$, whereas the CFS increased the secretion of TGF- $\beta 1$. However, in the presence of $E$. coli, both treatments restored the levels of TGF- $\beta$. The probiotic strain L. rhamnosus CNCM I- 4036 and its CFS were able to activate the Toll-like receptor signalling pathway, enhancing innate immunity. The two treatments induced gene transcription of TLR-9. Live L. rhamnosus activated the expression of $T L R-2$ and $T L R-4$ genes, whereas the CFS increased the expression of TLR-1 and TLR-5 genes. In response to the stimulation with probiotic/ CFS and $E$. coli, the expression of each gene tested was notably increased, with the exception of TNF- $\alpha$ and $N F K B I A$. In conclusion, the CFS exhibited an extraordinary ability to suppress the production of pro-inflammatory cytokines by DC, and may be used as an effective and safer alternative to live bacteria.

Key words: Cytokines: Dendritic cells: Gene expression: Probiotics: Toll-like receptors

Intestinal dendritic cells (DC) are central in controlling immune tolerance in the gastrointestinal tract ${ }^{(1)}$. DC capture antigens based on the expression of a wide variety of pattern-recognition receptors, including Toll-like receptors (TLR), nucleotide-binding oligomerisation domain-like receptors and C-type lectin receptors ${ }^{(2,3)}$, which play crucial roles in host recognition of both probiotics and foreign microorganisms ${ }^{(4)}$. These interactions induce signalling cascades and trigger a molecular response against the detected microorganism, including the production of immunomodulatory cytokines and chemokines, antimicrobial or cytoprotective factors and co-stimulatory molecules ${ }^{(5)}$. Bifidobacterium and Lactobacillus are the major commensal microbes of the gastrointestinal tract that are frequently used as probiotics ${ }^{(6)}$. In addition, recent reports have shown that probiotic treatment can modulate DC, as several Lactobacillus species regulated DC surface marker expression and cytokine production and maturation $^{(7-9)}$. However, the response of the immune system to probiotics remains controversial. Some probiotic strains modulate the production of cytokines by DC in vitro and induce a regulatory response, whereas others induce a pro-inflammatory response ${ }^{(10)}$. These strain-dependent effects are thought to be linked to specific interactions between bacteria and pattern-recognition receptors.

Escherichia coli is a common inhabitant of the human gut, where it acts as a harmless component of the normal flora. However, in some cases, it can be responsible for severe diarrhoea, especially in children ${ }^{(11)}$. Some studies have shown that probiotics have the potential to beneficially modulate the cytokine balance, promoting potent type- 1 immune responses

Abbreviations: CFS, cell-free culture supernatant; DC, dendritic cells; TGF, transforming growth factor; TLR, Toll-like receptor.

* Corresponding author: Dr M. Bermudez-Brito, fax +34 958 819132, email mbrito@correo.ugr.es 
or preventing immune dysregulation associated with specific T-cell polarisation ${ }^{(12)}$. For instance, probiotic bacteria alter the activation, expression and secretion of pro-inflammatory cytokines (such as TNF- $\alpha$ ) and chemokines (such as chemokine (C-X-C motif) ligand-8) caused by enteric pathogens ${ }^{(13)}$. However, these studies did not determine the mechanism(s) responsible for these probiotic immunomodulatory effects. Therefore, the aim of the present study was to (1) investigate the ability of Lactobacillus rhamnosus CNCM I-4036 and its cell-free culture supernatant (CFS) to activate human intestinal DC, (2) determine how they respond to pathogenic bacteria, specifically E. coli, and (3) elucidate the molecular mechanisms involved in these interactions. L. rhamnosus CNCM I-4036 was isolated from the faeces of breast-fed newborn infants, as described in a previous study ${ }^{(14)}$, and selected based on its probiotic properties, such as adhesion to intestinal mucus, sensitivity to antibiotics and resistance to gastrointestinal juices, biliary salts, $\mathrm{NaCl}$ and low $\mathrm{pH}$. The majority of studies that involved intestinal DC are based on mouse models, and research regarding human gut DC is limited, primarily due to methodological difficulties in studying human tissue and isolating human gut DC. Therefore, we used human DC that extend dendritic processes and that sample antigens in a manner similar to the way lamina propria DC sample luminal antigens.

\section{Materials and methods}

\section{Ethics statement}

The present study was approved by the University of Granada Ethical Committee. L. rhamnosus was obtained from the faeces of breast-fed newborn infants according to the protocol $^{(14)}$.

\section{Bacterial and supernatant preparation}

The isolated probiotic strain L. rhamnosus CNCM I-4036 $6^{(15)}$ was cultured in De Man-Rogosa-Sharpe broth medium (Oxoid) for $18-24 \mathrm{~h}$ at $37^{\circ} \mathrm{C}$ under anaerobic conditions. The CFS was obtained by centrifugation at $12000 \mathbf{g}$ for $10 \mathrm{~min}$, neutralised with $1 \mathrm{~m}-\mathrm{NaOH}$ to a $\mathrm{pH}$ of $7 \cdot 0$, concentrated 10-fold by lyophilisation and then sterilised by filtering through a $0.22 \mu \mathrm{m}$ filter (Minisart hydrophilic syringe filter; Sartorius Stedim Biotech $\mathrm{GmbH})$. The supernatant was added at a concentration of $7 \%(\mathrm{v} / \mathrm{v})$.

The Spanish Type Culture Collection (CECT) provided the pathogenic strains. The pathogens used in the present study included E. coli CECT 742, CECT 515 and CECT 729. The cultures were propagated aerobically in tryptone soya broth (Panreac Química).

In a previous study, we investigated the ability of $L$. rhamnosus CNCM I-4036 and its supernatant to inhibit the growth of different $E$. coli strains by monitoring bacterial growth at $37^{\circ} \mathrm{C}$ in tryptone soya and analysing the optical density at $620 \mathrm{~nm}$. Our data showed that live L. rhamnosus significantly inhibited the growth of enterotoxigenic E. coli (CECT 501 and CECT 515) and enteropathogenic E. coli (CECT 727 and
CECT 729). In the presence of non-neutralised supernatant, L. rhamnosus CNCM I-4036 inhibited the growth of these enteropathogens by 10-50\%; however, upon neutralisation, these effects were diminished except against E. coli CECT 742 (data not shown). Based on our previous study and in order to avoid changes in the media and DC death, live L. rhamnosus were incubated with E. coli CECT 515 and 729 , whereas its supernatant was incubated with $E$. coli 742 .

Before the experiments, pathogens were cultured at $37^{\circ} \mathrm{C}$ in tryptone soya broth for $8 \mathrm{~h}$ and then subcultured at a dilution of 1:500 in Roswell Park Memorial Institute-1640 medium (Sigma-Aldrich) containing 10\% fetal bovine serum (Gibco Invitrogen) at $37^{\circ} \mathrm{C}$ overnight.

\section{Cell preparation}

DC generated from umbilical cord blood $\mathrm{CD}_{3} 4^{+}$progenitor cells (haematopoietic stem cells) were supplied by MatTek Corporation $^{(16)}$. These cells were seeded onto twenty-fourwell plates in DC maintenance media (MatTek).

\section{Bacterial co-culture and dendritic cell stimulation}

DC were stimulated as described previously ${ }^{(8)}$. Briefly, DC were directly challenged for $4 \mathrm{~h}$ with live L. rhamnosus $\left(10^{7}\right.$ colony-forming units $\left./ \mathrm{ml}\right) / \mathrm{CFS}, \mathrm{E}$. coli $\left(10^{6}\right.$ colony-forming units $/ \mathrm{ml}$ ) or both. After incubation, the media were removed and replaced with fresh DC maintenance media. After $20 \mathrm{~h}$, culture supernatants were collected for cytokine analysis, and the cells were collected for RNA extraction. E. coli lipopolysaccharide $(20 \mathrm{ng} / \mathrm{ml}$; Sigma-Aldrich) was used as a positive control. Negative control cultures contained unstimulated DC.

\section{Cytokine and chemokine quantification in culture supernatants}

The amount of cytokines present in the supernatant was measured using MILLIplex ${ }^{\mathrm{TM}}$ immunoassays (Linco Research, Inc.) using the Luminex 200 system according to the manufacturer's instructions. IL-1 $\beta$, IL-6, IL-8, IL-10, IL-12p40, IL-12p70, TNF- $\alpha$, interferon- $\gamma$, monocyte chemotactic protein $1 /$ chemokine ( $\mathrm{C}-\mathrm{C}$ motif) ligand (CCL)-2, macrophage inflammatory protein-1 $\alpha /$ CCL-3, RANTES (regulated upon activation, normal T-cell expressed and presumably secreted)/CCL-5, macrophage-derived chemokine/CCL-22 and transforming growth factor (TGF)- $\beta$ were evaluated. Data are representative of three independent experiments.

\section{$R T$ reaction and $P C R$}

Inflammatory response in the bacteria-treated DC was examined using Human TLR Signaling Pathway PCR Arrays, as described previously by Bermudez-Brito et al. ${ }^{(8)}$. Each array comprised primer pairs that are specific to twenty genes related to TLR-mediated signalling pathways.

Briefly, DC were lysed and total RNA was extracted using the RNAqueous Kit (Ambion) and Turbo DNase treatment 
(Ambion), according to the manufacturer's recommendations. RNA quality was verified with a Model 2100 Bioanalyzer (Agilent), and RNA concentration was determined using a RediPlate 96 RiboGreen RNA Quantitation Kit (Gibco, Invitrogen).

Gene expression was analysed using $\mathrm{RT}^{2}$ Profiler PCR Array Data Analysis software (version 3.4; SABiosciences). Changes in expression or activity levels are expressed as fold changes. Results represent the fold increase relative to the control samples (untreated DC).

\section{Statistical analysis}

All data are expressed as means with their standard errors. Statistical analyses were performed using the Mann-Whitney
$U$ test. Statistical calculations were made using NCSS 2007 software. $P<0 \cdot 05$ was considered as statistically significant.

\section{Results}

Lactobacillus rhamnosus CNCM I-4036 and its supernatant reduce pro-inflammatory cytokine production in Escherichia coli-challenged human dendritic cells

The immunomodulatory effects of live L. rhamnosus CNCM I-4036 and its supernatant were determined in vitro using human DC. The addition of E. coli (E. coli CECT 742, E. coli CECT 515 or E. coli CECT 729) or lipopolysaccharides induced significant changes in the profile of cytokines in DC.

(a)

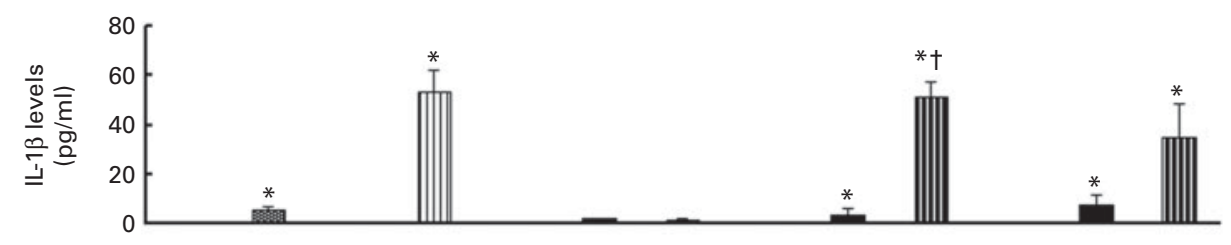

(b)

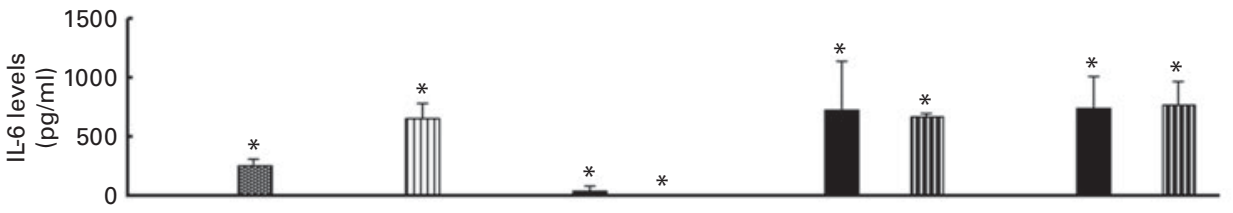

(c)

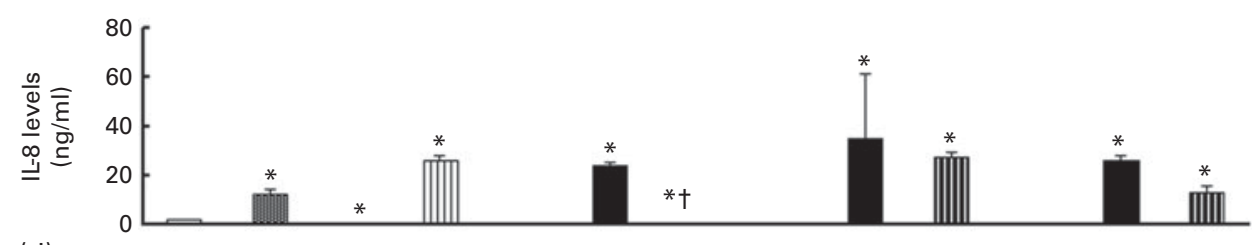

(d)

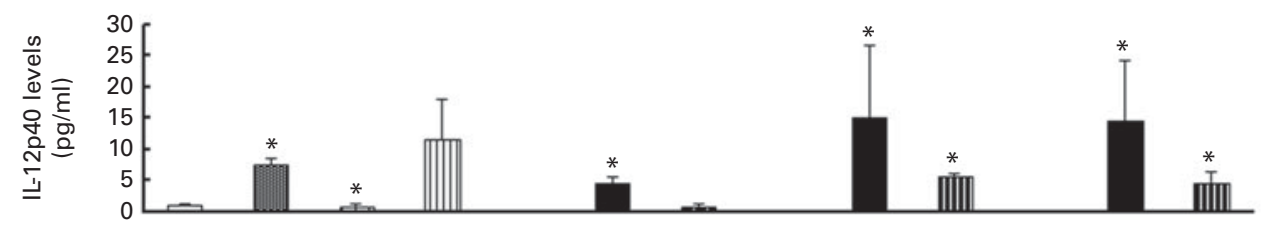

(e)

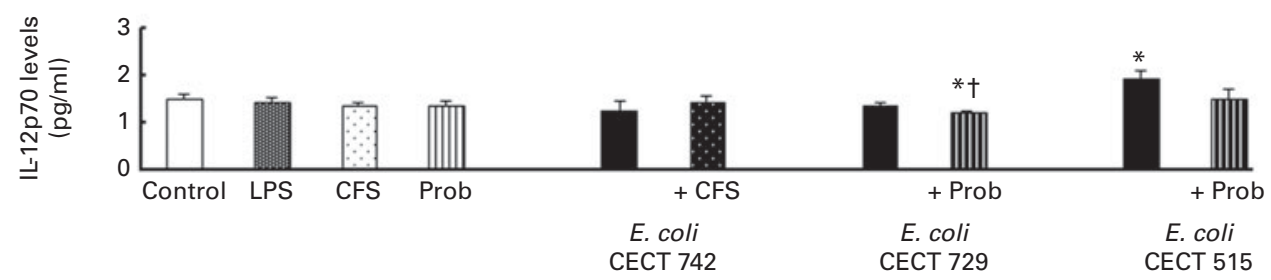

Fig. 1. Pro-inflammatory cytokine production in dendritic cells (DC) after exposure to Lactobacillus rhamnosus, Escherichia coli or both. DC incubated with different bacterial strains demonstrate distinct profiles of IL-1 $\beta$ (a), IL-6 (b), IL-8 (c), IL-12p40 (d) and IL-12p70 (e) production. The levels of these cytokines were measured after DC were incubated for $4 \mathrm{~h}$ with probiotics (live bacteria (Prob) or supernatant), pathogen or both and then further incubated for $20 \mathrm{~h}$ in media containing antibiotics. Culture supernatants were collected, and cytokine levels were assessed by immunoassay. Values are means of three different experiments, with their standard errors represented by vertical bars. ${ }^{*}$ Mean value was significantly different from that of untreated $\mathrm{DC}$ (control) $(P<0.05)$. † Mean value was significantly different from that of pathogen-treated cells $(P<0.05)$. LPS, lipopolysaccharide; CFS, cell-free culture supernatant. 
The treatments with live L. rhamnosus CNCM I-4036 and its supernatant markedly increased the levels of proinflammatory cytokines such as IL-6, IL-8, IL-12p40 (Fig. 1) and TNF- $\alpha$ (Fig. 2), compared with the untreated DC (control). In DC, L. rhamnosus CNCM I-4036 and its CFS exerted differential effects on the induction of cytokines.

Interestingly, in the absence of any pathogen, the CFS by itself did not affect the secretion of pro-inflammatory cytokines or chemokines. Moreover, CFS treatment decreased the secretion of IL-8, IL-12p40 (Fig. 1) and all the chemokines tested compared with the untreated DC (control) (Fig. 3). In response to the stimulation with $L$. rhamnosus CNCM I-4036, supernatant and E. coli CECT 742, there was a decrease in the release of pro-inflammatory cytokines such as IL-8 and TNF- $\alpha$ (Figs. 1 and 2) and chemokines such as monocyte chemotactic protein-1 and RANTES (Fig. 3). Furthermore, in the presence of $E$. coli CECT 742, CFS induced the secretion of TGF- $\beta 1$ (Fig. 2).

In general, as shown in Figs. 1 and 2, co-treatment with live L. rhamnosus CNCM I-4036 and E. coli had little effect on cytokine secretion, with the exception of a significant decrease in the levels of TNF- $\alpha$ and monocyte chemotactic protein-1 (Fig. 3). In contrast, in the absence of pathogens, live L. rhamnosus CNCM I-4036 increased the overall production of cytokines and chemokines, including that of IL-1 $\beta$ and IL- 6 (Figs. 1-3). In the culture media, the analyses of TGF- $\beta$ clearly showed that live L. rhamnosus CNCM I-4036 potently induced the production of TGF- $\beta 1$ and TGF- $\beta 2$ (Fig. 2). We did not detect TGF- $\beta 3$ under any of the treatment conditions (data not shown).
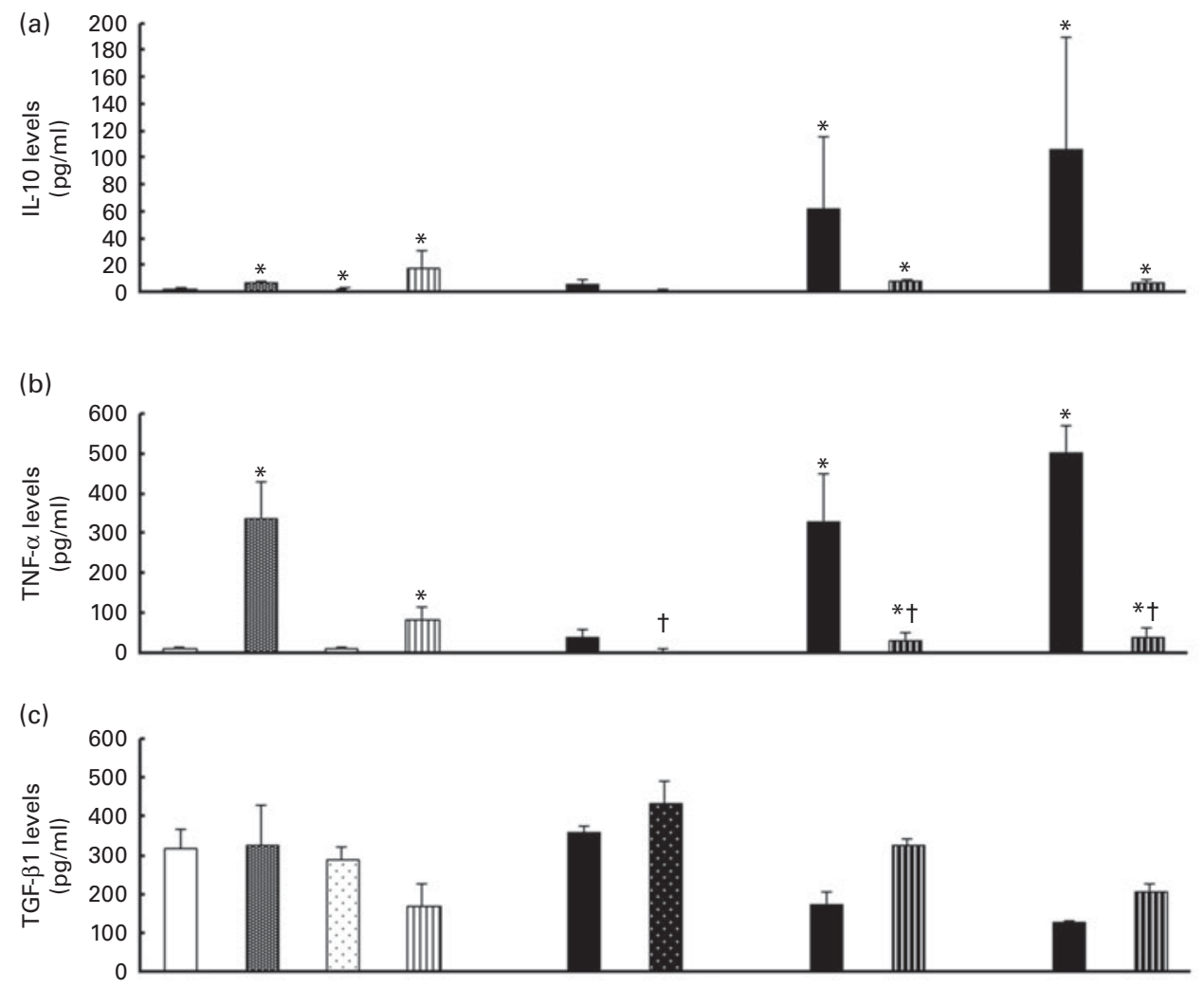

(d)

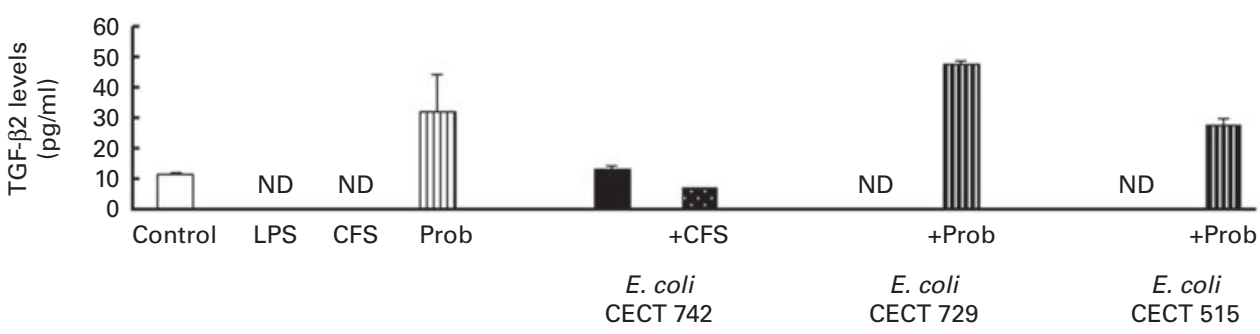

Fig. 2. Anti-inflammatory cytokine production in dendritic cells (DC) after exposure to Lactobacillus rhamnosus, Escherichia coli or both. DC incubated with different bacterial strains demonstrate distinct profiles of IL-10 (a), TNF- $\alpha$ (b), transforming growth factor (TGF)- $\beta 1$ (c) and TGF- $\beta 2$ (d) production. The levels of these cytokines were measured after DC were incubated for $4 \mathrm{~h}$ with probiotics (live bacteria (Prob) or supernatant), pathogen or both and then further incubated for $20 \mathrm{~h}$ in media containing antibiotics. Culture supernatants were collected, and cytokine levels were assessed by immunoassay. Values are means of three different experiments, with their standard errors represented by vertical bars. * Mean value was significantly different from that of untreated $D C$ (control) $(P<0.05)$. $\dagger$ Mean value was significantly different from that of pathogen-treated cells $(P<0.05)$. LPS, lipopolysaccharide; CFS, cell-free culture supernatant; ND, not determined. 


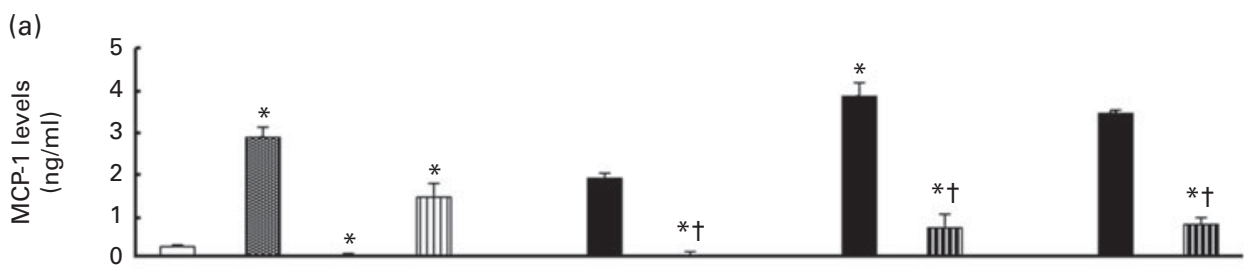

(b)
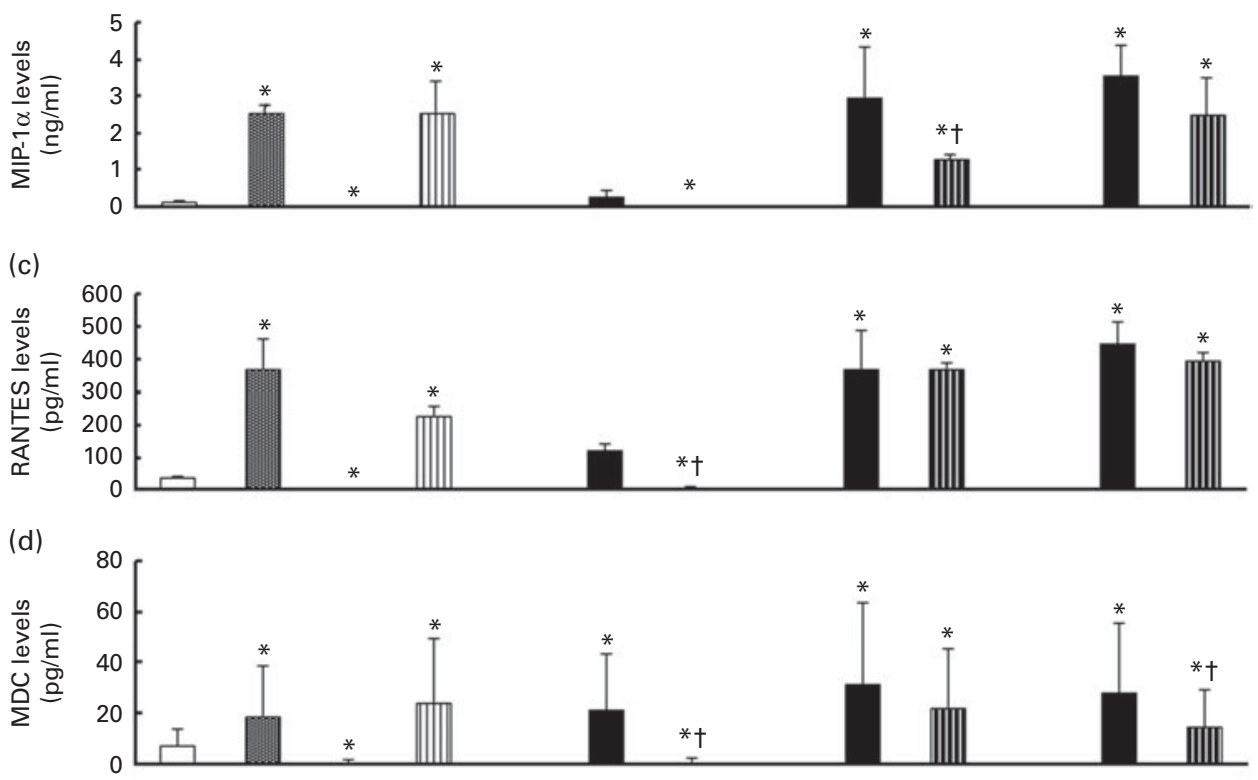

(e)

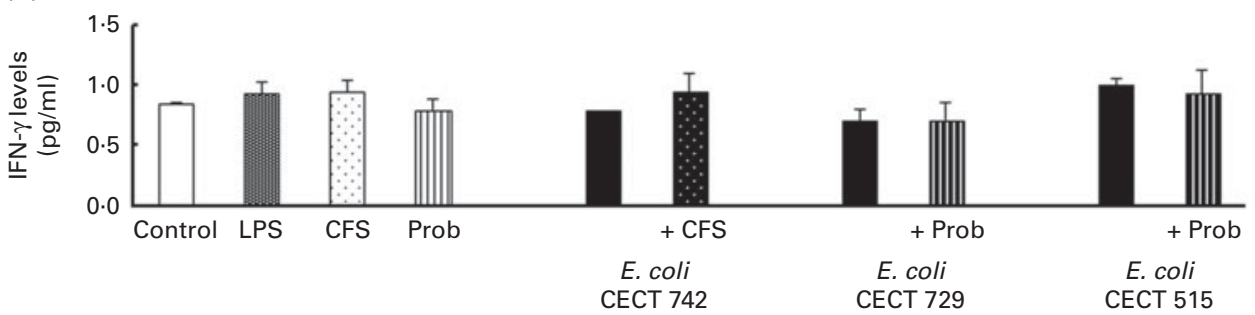

Fig. 3. Release of chemokines in dendritic cells (DC) after exposure to Lactobacillus rhamnosus, Escherichia coli or both. DC incubated with different bacterial strains demonstrate distinct profiles of monocyte chemotactic protein-1 (MCP-1)/chemokine (C-C motif) ligand (CCL)-2 (a), macrophage inflammatory protein-1 $\alpha$ $(\mathrm{MIP}-1 \alpha) / C C L-3$ (b), regulated upon activation, normal T-cell expressed and presumably secreted (RANTES)/CCL-5 (c), macrophage-derived chemokine (MDC)/CCL-22 (d) and interferon (IFN)- $\gamma$ (e) production. The levels of these chemokines were measured after DC were incubated for $4 \mathrm{~h}$ with probiotics (live bacteria (Prob) or supernatant), pathogens or both and then further incubated for $20 \mathrm{~h}$ in media containing antibiotics. Culture supernatants were collected, and cytokine levels were assessed by immunoassay. Values are means of three different experiments, with their standard errors represented by vertical bars. * Mean value was significantly different from that of untreated DC (control) $(P<0.05)$. † Mean value was significantly different from that of pathogen-treated cells $(P<0.05)$. LPS, lipopolysaccharide; CFS, cell-free culture supernatant.

\section{Lactobacillus rhamnosus CNCM I-4036 induces Toll-like receptor signalling pathway gene expression in human dendritic cells}

We also evaluated TLR signalling pathway gene expression. As shown in Figs. 4-7, exposure of DC to the different strains of $E$. coli for $4 \mathrm{~h}$ resulted in similar TLR expression patterns. The E. coli strains upregulated the gene expression of TLR-1, TLR-2 and TLR-4 (Fig. 4). Notably, TLR-9 was overexpressed in the treated DC (Fig. 5), and increased the gene expression of IL-1 receptor-associated kinase-4 (IRAK-4), c-Jun aminoterminal kinase $(J N K)$, interferon regulatory factor $(I R F-3)$ and $I L-1 O$ (Figs. 5-7).
The probiotic strain L. rhamnosus CNCM I- 4036 and its CFS similarly affected TLR pathway gene expression (Figs. 4-7). The probiotic strain alone (live L. rhamnosus) stimulated the gene expression of TLR-2 and TLR- 4 , whereas its supernatant significantly increased the expression of TLR-1 and TLR-5 (Fig. 4). Both treatments induced strong and sustained transcription of TLR-9 (Fig. 5).

Interestingly, the gene expression of TLR-1, TLR-2, TLR-5 and TLR-9 was notably increased in response to the stimulation with CFS and E. coli (Figs. 4 and 5). Conversely, DC exposure to live probiotic and enteropathogens downregulated the gene expression of $T L R-3$, but increased the 
(a)

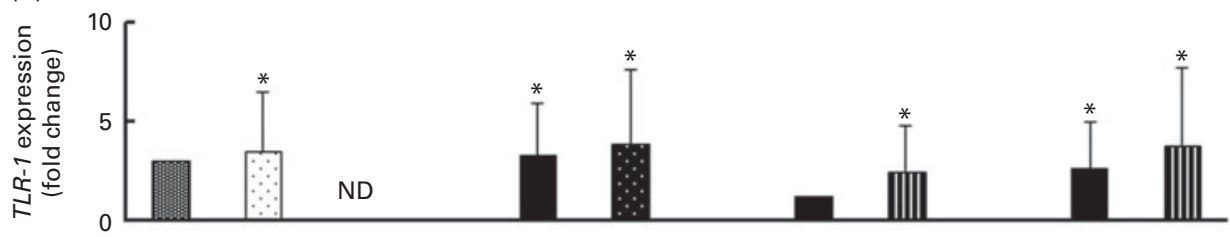

(b)

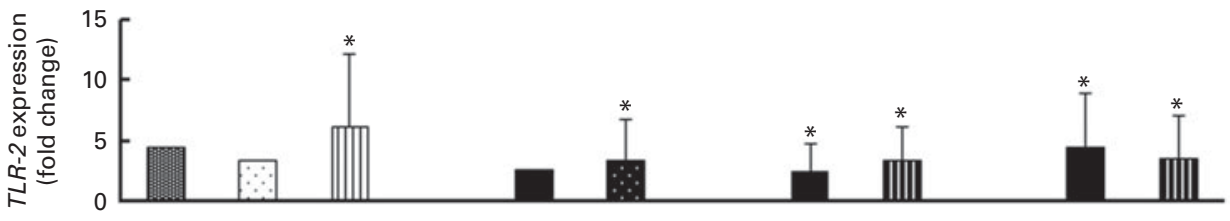

(c)

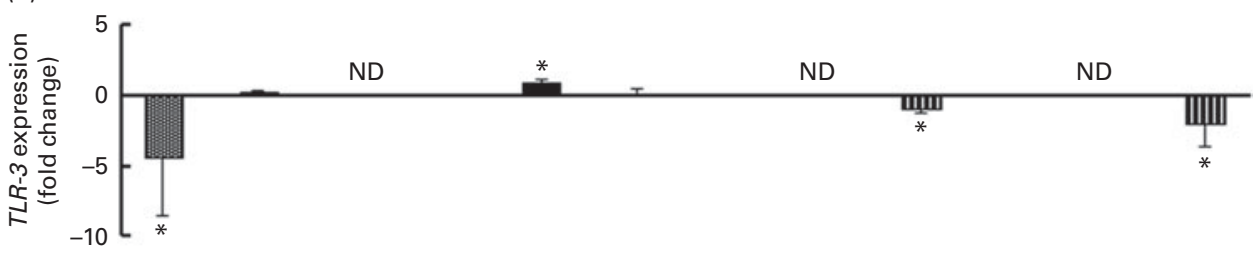

(d)
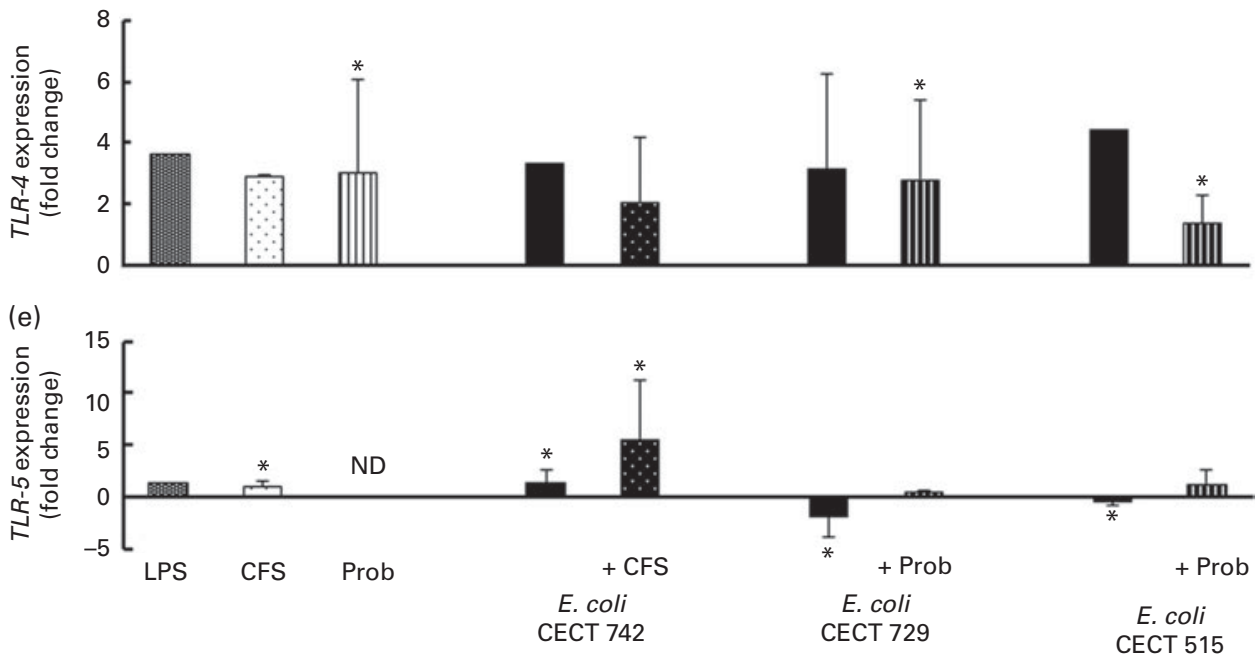

Fig. 4. Comparison of Toll-like receptor-1 (TLR-1) (a), TLR-2 (b), TLR-3 (c), TLR-4 (d) and TLR-5 (e) expression in dendritic cells (DC) in the presence of probiotic (Prob)/supernatant, Escherichia coli or both. The fold change represents the ratio of the expression in the treated DC compared with the control cells. Values are means of three different experiments, with their standard errors represented by vertical bars. ${ }^{*}$ Mean value was significantly different from that of untreated DC (control) $(P<0.05)$. ND, not determined; LPS, lipopolysaccharide; CFS, cell-free culture supernatant.

expression of TLR-4 (Fig. 4). Interestingly, the gene expression of $T L R-5$ was increased in response to the stimulation with CFS and E. coli (Fig. 4).

As shown in Figs. 4-7, exposure of DC to the probiotic strain L. rhamnosus CNCM I-4036 or its CFS with E. coli activated the TLR signalling pathway, and the expression of all the genes tested was increased.

Interestingly, upon stimulation with CFS or live probiotic plus $E$. coli, the gene expression of Toll-interacting protein (TOLLIP; Fig. 5) and caspase-8 (CASP-8; Fig. 6) was increased. A similar effect was observed for $I R F-3$ (Fig. 6) and TANKbinding kinase-1 (TBK-1; Fig. 7).
We also observed differences between the treatments. In the presence of $E$. coli, the CFS induced the expression of $J N K$, whereas its expression was decreased by L. rhamnosus (Fig. 6). In addition, the stimulation with CFS and E. coli decreased the gene expression of NF-кB inhibitor $\alpha$ (NFKBIA), whereas it was increased by the live probiotic strain (Fig. 7).

\section{Discussion}

Several studies have suggested that the in vitro peripheral blood mononuclear cell model is a useful screening tool to identify characteristic traits of probiotic strains to select 
(a)

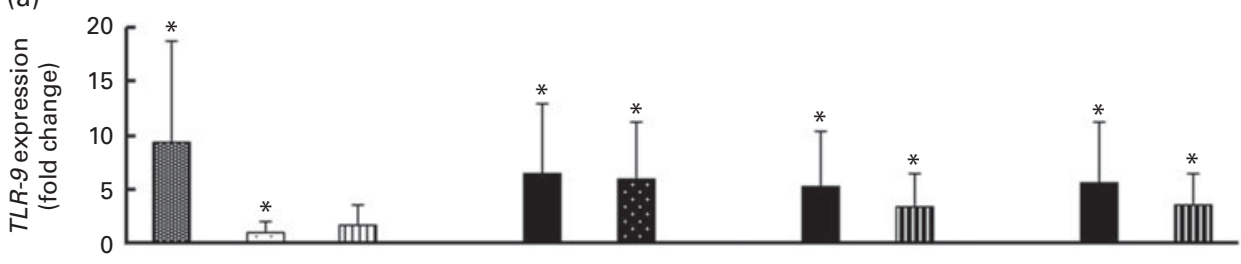

(b)

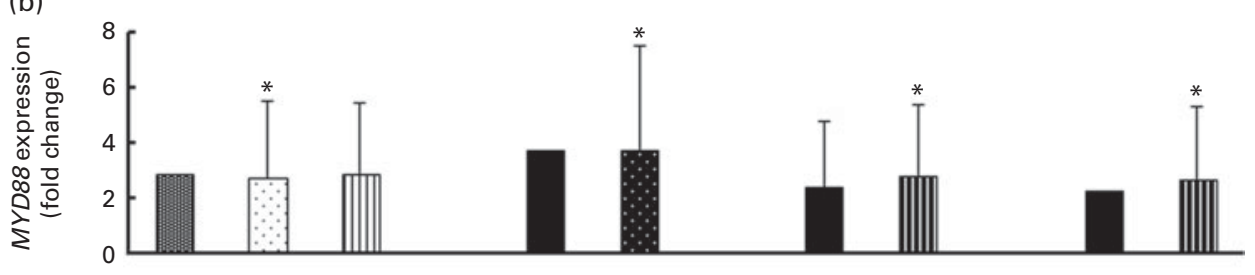

(c)

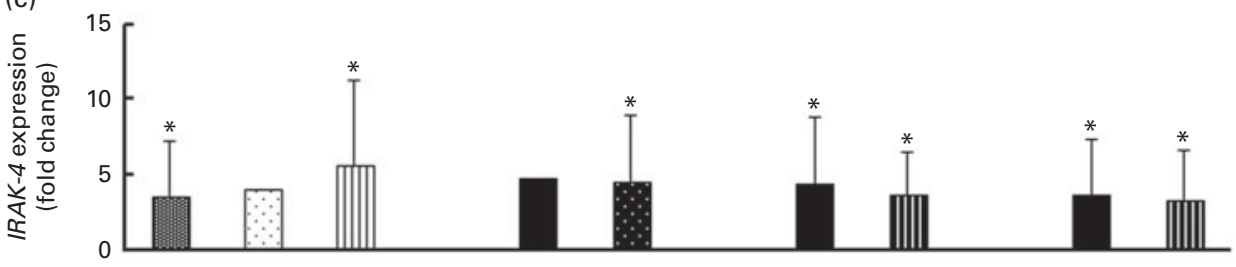

(d)
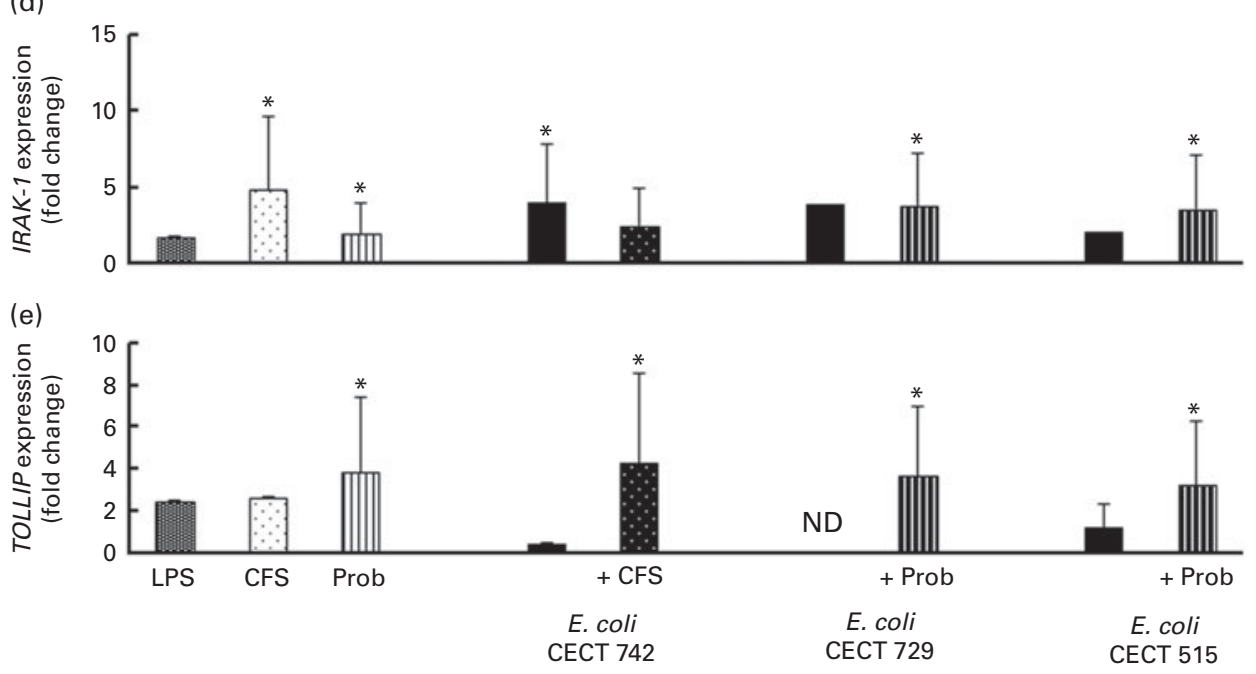

Fig. 5. Comparison of Toll-like receptor 9 (TLR-9) (a), myeloid differentiation factor 88 (MYD88) (b), IL-1 receptor-associated kinase 4 (IRAK-4) (c), IRAK-1 (d) and Toll-interacting protein (TOLLIP) (e) expression in dendritic cells (DC) in the presence of probiotic (Prob)/supernatant, Escherichia coli or both. The fold change represents the ratio of the expression in the treated DC relative to the control cells. Values are means of three different experiments, with their standard errors represented by vertical bars. * Mean value was significantly different from that of untreated DC (control) $(P<0.05)$. LPS, lipopolysaccharide; CFS, cell-free culture supernatant; ND, not determined.

probiotic strains for clinical trials ${ }^{(17-21)}$. However, these peripheral blood mononuclear cells and murine DC are quite different from human gut DC. Therefore, we employed an in vitro culture system using human DC that were harvested from umbilical cord blood $\mathrm{CD}_{3} 4^{+}$progenitor cells (and were similar to lamina propria gut DC) to study the immunological effects and anti-inflammatory properties of $L$. rhamnosus CNCM I-4036, a potentially probiotic strain isolated from the faeces of breast-fed newborn infants, and its supernatant.

Differences in the induction of the cytokine profile in human DC challenged with E. coli were observed between L. rhamnosus CNCM I-4036 and its supernatant. Specifically, in the presence of E. coli, L. rhamnosus CNCM I-4036 and its CFS reduced the production of pro-inflammatory cytokines and chemokines. However, the CFS did not induce the secretion of IL-12p40. In addition, in the absence of enteropathogens, the stimulation with CFS did not remarkably alter DC-produced cytokines. Recently, our group demonstrated a similar effect for L. paracasei CNCM I-4034(8) and Bifidobacterium breve CNCM I-4035 supernatants ${ }^{(22)}$; the CFS from these probiotic strains exhibited anti-inflammatory behaviour by decreasing the production of inflammatory cytokines. Taken together, these data suggest that the probiotic strains L. paracasei CNCM I-4034, B. breve CNCM I-4035 and L. rhamnosus CNCM I-4036 release unknown factors capable of modulating DC cytokine production. One possible mechanism that 
(a)

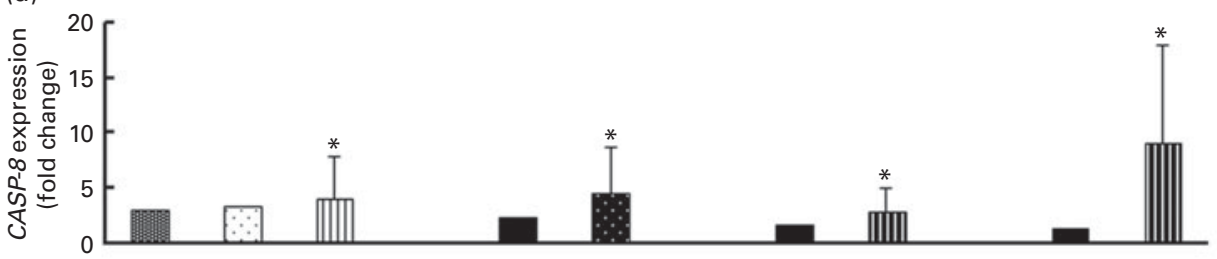

(b)

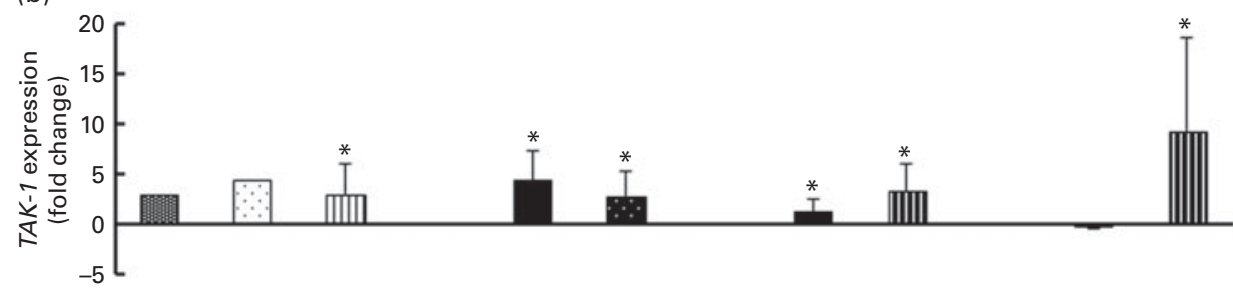

(c)

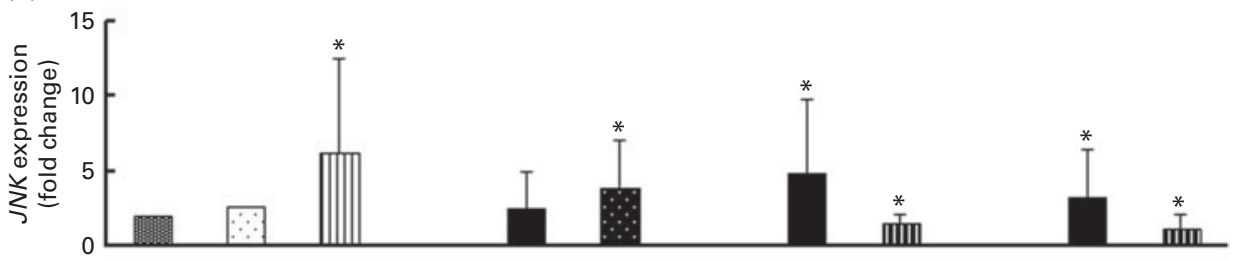

(d)

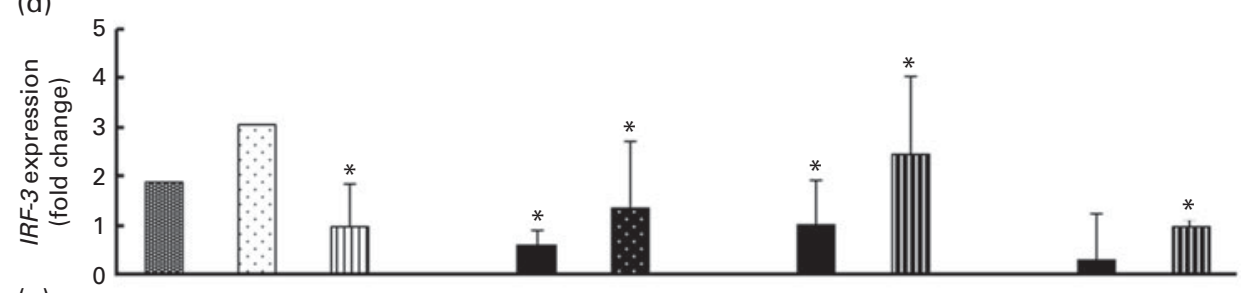

(e)

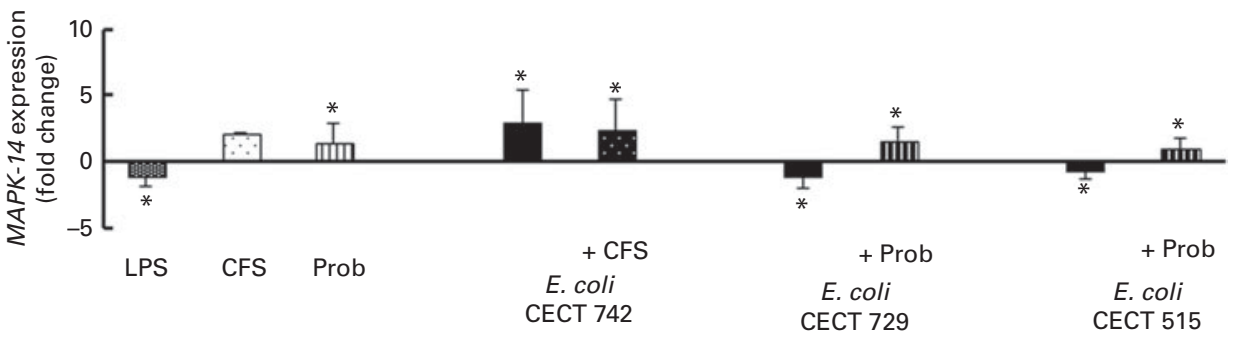

Fig. 6. Comparison of caspase-8 (CASP-8) (a), TGF- $\beta$-activated kinase-1 (TAK-1) (b), c-Jun amino-terminal kinase (JNK) (c), interferon regulatory factor-3 (IRF-3) (d) and mitogen-activated protein kinase-14 (MAPK-14) (e) expression in dendritic cells (DC) in the presence of probiotic (Prob)/supernatant, Escherichia coli or both. The fold change represents the ratio of the expression in the treated DC compared with the control cells. Values are means of three different experiments, with their standard errors represented by vertical bars. * Mean value was significantly different from that of untreated DC (control) $(P<0.05)$. LPS, lipopolysaccharide; CFS, cell-free culture supernatant.

could explain these immunomodulatory properties is the production and secretion of inhibitory metabolites in the media by probiotics. As a reflection of the differences between live probiotic bacteria and their supernatants, it is important to take into account that the supernatants were concentrated three times, so any substance released in the media would have a 10-fold effect compared with the live probiotic. In addition, these compounds that are present in different concentrations in live L. rhamnosus and its supernatant, but with the same origin, could explain the similar effects on TLR signalling genes. In contrast, the identification of proteins predicted to be involved in such interactions can pave the way towards well-targeted studies of the protein-mediated contacts between bacteria and the host, with the goal to enhance the understanding of the mode of action of probiotic bacteria. Ongoing work in our laboratory to unravel the proteins involved in these interactions reveals that these bacteria secrete a number of proteins that are predicted to play an important role in immunomodulation of the host immune system. Of special interest is the identification of three proteins excreted by L. rhamnosus CNCM I-4036 that may have potential implications in the direct interaction 

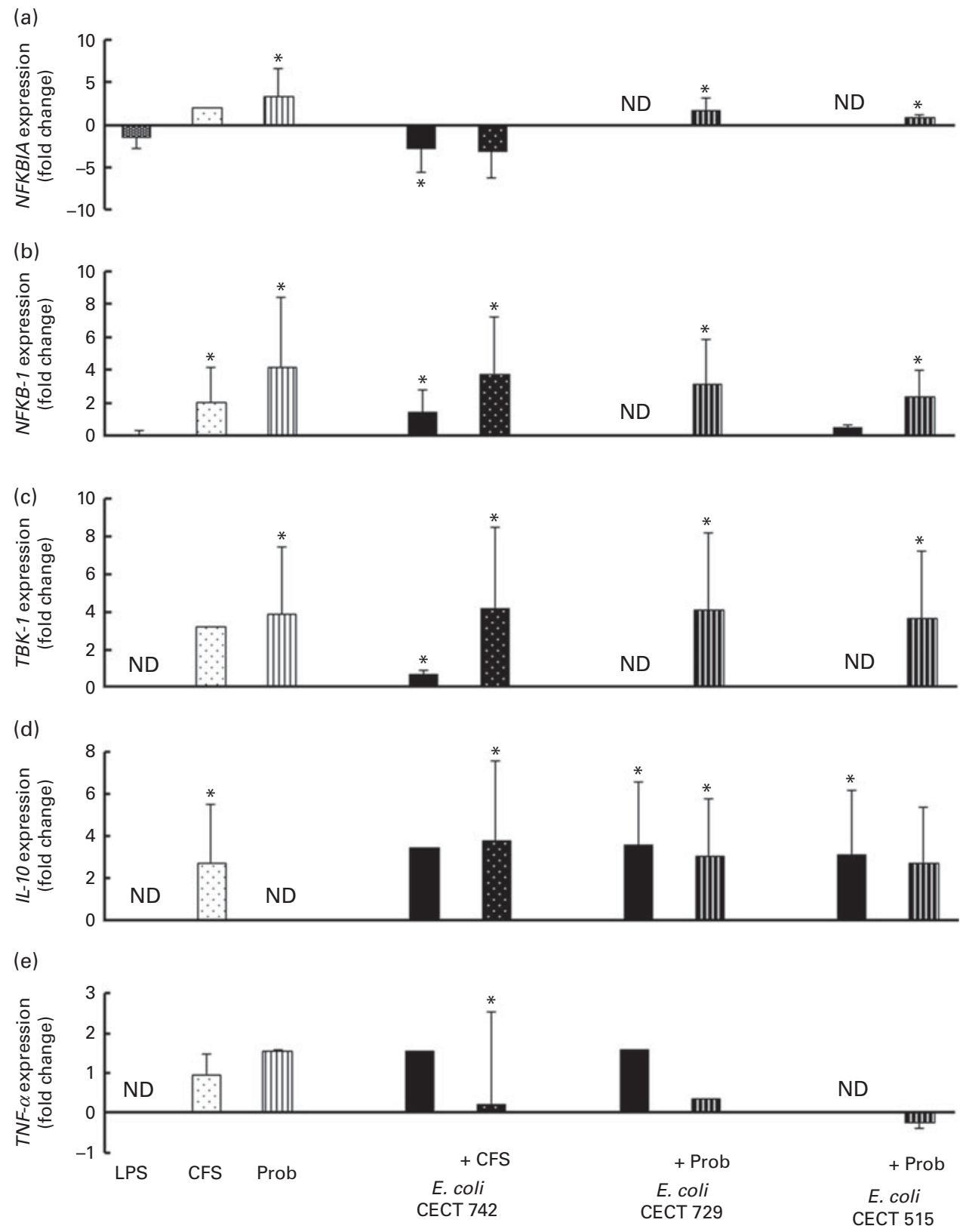

Fig. 7. Comparison of NF-KB inhibitor $\alpha$ (NFKBIA) (a), NFKB-1 (b), TANK-binding kinase-1 (TBK-1) (c), IL-10 (d) and TNF- $\alpha$ (e) expression in dendritic cells (DC) in the presence of probiotic (Prob)/supernatant, Escherichia coli or both. The fold change represents the ratio of the expression in the treated DC compared with the control cells. Values are means of three different experiments, with their standard errors represented by vertical bars. * Mean value was significantly different from that of untreated DC (control) $(P<0.05)$. ND, not determined; LPS, lipopolysaccharide; CFS, cell-free culture supernatant.

with the host, i.e. attachment to mucin and intestinal cells (M Fernández, S Juárez, S Ciordia, JM Vieites, JP Albar, A Gil and $M$ Ferrer, unpublished results).

Interestingly, in the presence of E. coli, L. rhamnosus CNCM I-4036 promoted the production of TGF- $\beta 1$ and TGF- $\beta 2$, whereas the supernatant increased the secretion of TGF- $\beta 1$. This effect was also observed with $L$. paracasei CNCM I-4034 and its supernatant ${ }^{(8)}$. TGF- $\beta 1$ is an important inhibitor of the synthesis of pro-inflammatory cytokines ${ }^{(23)}$. This result indicates that the secretion of TGF- $\beta 1$ after DC probiotic stimulation may be a mechanism by which these Lactobacillus strains exert anti-inflammatory effects.
The probiotic strain L. rhamnosus CNCM I-4036 did not reduce the secretion of IL- 6 , and, in fact, IL- 6 induction by the probiotic strain was observed in the absence of any pathogen. IL-6 promotes terminal differentiation of B cells into plasma cells, and furthermore, it has been found to be able to polarise naive $\mathrm{CD}^{+}{ }^{+} \mathrm{T}$ cells to effector Th2 cells. The relatively strong ability to induce the production of IL- 6 that was observed for all the Lactobacillus strains is consistent with the general finding that many species of lactic acid bacteria induce IL- 6 production and enhance intestinal IgA responses ${ }^{(9)}$.

In the absence of $E$. coli, the probiotic strain induced the production of IL-1 $\beta$, IL- 6 , IL- 8 , IL-12 and TNF- $\alpha$, whereas the 
supernatant suppressed the release of these pro-inflammatory cytokines. These results are in accordance with the earlier findings demonstrating that only intact bacteria induce appreciable amounts of IL-12 ${ }^{(24)}$. Because expression of $I L-8$, $T N F-\alpha$ and $I L-6$ is regulated by $N F-\kappa B^{(25)}$, these results suggest that live L. rhamnosus CNCM I-4036 may enhance NF-кB-mediated innate immunity rather than suppression, as reported by Pagnini et al. ${ }^{(26)}$. Furthermore, NF- $\mathrm{BB}$ reportedly plays a beneficial role in epithelial cells. The activation of $\mathrm{NF}-\kappa \mathrm{B}$ similarly protects against the development of systemic inflammation $^{(26)}$.

The probiotic L. rhamnosus CNCM I-4036 and its supernatant stimulated the gene expression of TLR-9 in the presence or absence of $E$. coli, indicating that this bacterial strain contains immunostimulatory double-stranded DNA motifs with unmethylated CpG sequences ${ }^{(27)}$, which are powerful activators of the innate immune system. There are three types of $\mathrm{CpG}$ oligodeoxynucleotides, namely $\mathrm{CpG}-\mathrm{A}$, CpG-B and CpG-C (a mixture of CpG-A and CpG-B). CpG oligodeoxynucleotide strongly activates conventional DC to produce pro-inflammatory IL- 6 and IL-12 ${ }^{(28)}$. It is known that CpG oligodeoxynucleotide activates its receptor TLR-9, which in turn recruits the adaptor protein myeloid differentiation factor 88 and IRAK4, leading to the phosphorylation and activation of IRAK $4^{(29)}$. In addition, it has been reported that Lactobacillus-mediated cytokine induction is strongly dependent on TLR-9 ${ }^{(27)}$.

As expected, DC expressed TLR-2 in response to the stimulation with L. rhamnosus. TLR-2 has been shown to recognise peptidoglycan, which is the main component of Grampositive bacteria such as Lactobacillus. In addition, lactobacilli have been shown to mediate their effects via TLR-2 ${ }^{(24,30)}$, possibly via lipoteichoic acid, a TLR-2 agonist and one of the main immunostimulatory components of Lactobacillus strains. Furthermore, in vitro and ex vivo studies have demonstrated that some immunostimulatory lactobacilli interact with intestinal epithelial cells and induce the release of IL-6 through an interaction with TLR-2 ${ }^{(30)}$. Interestingly, in the presence of $E$. coli and the supernatant, DC up-regulated the expression of $T L R-5$, and a previous study suggested that expression of this TLR may prevent immune responses against commensal Gram-negative bacteria ${ }^{(31)}$.

Taken together, the present results demonstrate that exposure of intestinal-like human DC to the probiotic strain L. rhamnosus CNCM I-4036 or its CFS with E. coli activates the TLR signalling pathway, as the expression of each gene tested was increased, with the exception of $T N F-\alpha$ and NFKBIA. In the absence of pathogens, the probiotic strain L. rhamnosus CNCM I-4036 and its CFS increased the expression of $N F K B I A$, which is one of the most important negative regulators of $N F-\kappa B$ and acts by sequestering this transcription factor in the cytoplasm and preventing its nuclear translocation. These data reveal the anti-inflammatory character of L. rhamnosus CNCM I-4036 and its CFS. In addition, it has been reported that L. salivarius UCC118 induces the expression of this gene in Caco- 2 cells ${ }^{(32)}$.

In conclusion, the present results indicate that some probiotic strains such as L. rhamnosus CNCM I-4036 are immunostimulatory, whereas other strains are immunomodulatory ${ }^{(8)}$, which demonstrates that generalisations concerning the biological effects and potential health benefits of probiotics should be avoided ${ }^{(33)}$ and implies that specific probiotic strains interact uniquely with DC to induce strain-specific effects $^{(34)}$

Finally, L. rhamnosus CNCM I-4036-secreted products sensed by host pattern-recognition receptors exhibited an extraordinary ability to suppress the production of pro-inflammatory cytokines by DC and may be used as an effective and safer alternative to live bacteria.

\section{Acknowledgements}

The present study was supported by Hero Group, Spain S.A. through a contract (no. 3143) signed with the Fundación General Universidad de Granada-Empresa and co-sponsored by a CDTI project, a public entity of the Ministry of Economy and Competitiveness of Spanish Government. C. G.-L. has a postdoctoral fellowship from the Plan Propio of the University of Granada. Hero Group, Spain S.A. had no role in the design, analysis or writing of this article.

The authors' contributions are as follows: A. G. and C. G.-L. conceived and designed the experiments; M. B.-B. and S. M. Q. performed the experiments; M. B.-B. analysed the data; F. R. contributed reagents/materials/analysis tools; M. B.-B. and A. G. wrote the manuscript.

F. R. is a member of Hero Global Technology Centre for Infant Nutrition, Hero Group, Spain S.A. The sponsor had no role in the biological sample analysis, statistical analysis or data interpretation. None of the other authors has any conflict of interest.

\section{References}

1. Bernardo D, Sánchez B, Al-Hassi HO, et al. (2012) Microbiota/host crosstalk biomarkers: regulatory response of human intestinal dendritic cells exposed to Lactobacillus extracellular encrypted peptide. PLOS ONE 7, e36262.

2. Dzopalic T, Rajkovic I, Dragicevic A, et al. (2012) The response of human dendritic cells to co-ligation of patternrecognition receptors. Immunol Res 52, 20-33.

3. Bermudez-Brito M, Plaza-Díaz J, Muñoz-Quezada S, et al. (2012) Mechanisms of action of probiotics. Ann Nutr Metab 61, 160-174.

4. Meijerink M \& Wells JM (2010) Probiotic modulation of dendritic cells and $\mathrm{T}$ cell responses in the intestine. Benef Microbes 1, 317-326.

5. Lebeer S, Vanderleyden J \& De Keersmaecker SC (2010) Host interactions of probiotic bacterial surface molecules: comparison with commensals and pathogens. Nat Rev Microbiol 8, 171-184.

6. Rizzello V, Bonaccorsi I, Dongarrà ML, et al. (2012) Role of natural killer and dendritic cell crosstalk in immunomodulation by commensal bacteria probiotics. J Biomed Biotechnol 2011, 473097.

7. Kim YG, Ohta T, Takahashi T, et al. (2006) Probiotic Lactobacillus casei activates innate immunity via NF-kappa B and p38 MAP kinase signaling pathways. Microbes Infect $\mathbf{8}$, 994-1005. 
8. Bermudez-Brito M, Muñoz-Quezada S, Gomez-Llorente C, et al. (2012) Human intestinal dendritic cells decrease cytokine release against Salmonella infection in the presence of Lactobacillus paracasei upon TLR activation. PLOS ONE 7, e43197.

9. Christensen HR, Frøkiaer H \& Pestka JJ (2002) Lactobacilli differentially modulate expression of cytokines and maturation surface markers in murine dendritic cells. J Immunol 168, 171-178.

10. Evrard B, Coudeyras S, Dosgilbert A, et al. (2011) Dosedependent immunomodulation of human dendritic cells by the probiotic Lactobacillus rhamnosus Lcr35. PLOS ONE $\mathbf{6}$, e18735.

11. Santona S, Diaz N, Fiori PL, et al. (2013) Genotypic and phenotypic features of enteropathogenic Escherichia coli isolated in industrialized and developing countries. J Infect Dev Ctries 7, 214-219.

12. Bermudez-Brito M, Plaza-Díaz J, Fontana L, et al. (2013) In vitro cell and tissue models for studying host-microbe interactions: a review. Br J Nutr 109, S35-S50.

13. Gareau MG, Ho NK, Brenner D, et al. (2011) Enterohaemorrhagic, but not enteropathogenic, Escherichia coli infection of epithelial cells disrupts signalling responses to tumour necrosis factor-alpha. Microbiology 157, Pt 10, 2963-2973.

14. Vieites Fernández JM, Muñoz Quezada S, Llamas Company I, et al. (2010) Aislamiento, identificación y caracterización de cepas con actividad prebiótica a partir de heces de lactantes alimentados exclusivamente con leche materna. PCT AX090006WO.

15. Muñoz-Quezada S, Chenoll E, Vieites-Fernández JM, et al. (2013) Isolation, identification and characterisation of three novel probiotic strains (Lactobacillus paracasei CNCM I-4034, Bifidobacterium breve CNCM I-4035 and Lactobacillus rhamnosus CNCM I-4036) from the faeces of exclusively breast-fed infants. BrJ Nutr 109, Suppl. 2, S51-S62.

16. Ayehunie S, Snell M, Child M, et al. (2009) A plasmacytoid dendritic cell (CD123+/CD11c-) based assay system to predict contact allergenicity of chemicals. Toxicology 264, 1-9.

17. Helwig U, Lammers KM, Rizzello F, et al. (2006) Lactobacilli, bifidobacteria and $E$. coli nissle induce pro- and antiinflammatory cytokines in peripheral blood mononuclear cells. World J Gastroenterol 12, 5978-5986.

18. Medina M, Izquierdo E, Ennahar S, et al. (2007) Differential immunomodulatory properties of Bifidobacterium longum strains: relevance to probiotic selection and clinical applications. Clin Exp Immunol 150, 531-538.

19. Imaoka A, Shima T, Kato K, et al. (2008) Anti-inflammatory activity of probiotic Bifidobacterium: enhancement of IL-10 production in peripheral blood mononuclear cells from ulcerative colitis patients and inhibition of IL- 8 secretion in HT-29 cells. World J Gastroenterol 14, 2511-2516.

20. Dong H, Rowland I, Tuohy KM, et al. (2010) Selective effects of Lactobacillus casei Shirota on $\mathrm{T}$ cell activation, natural killer cell activity and cytokine production. Clin Exp Immunol 161, 378-388.
21. Pérez-Cano FJ, Dong H \& Yaqoob P (2010) In vitro immunomodulatory activity of Lactobacillus fermentum CECT5716 and Lactobacillus salivarius CECT5713: two probiotic strains isolated from human breast milk. Immunobiology $\mathbf{2 1 5}$, 996-1004.

22. Bermudez-Brito M, Muñoz-Quezada S, Gomez-Llorente C, et al. (2013) Cell-free culture supernatant of Bifidobacterium breve CNCM I-4035 decreases pro-inflammatory cytokines in human dendritic cells challenged with Salmonella typhi through TLR activation. PLOS ONE 8, e59370.

23. Parlesak A, Haller D, Brinz S, et al. (2004) Modulation of cytokine release by differentiated CACO-2 cells in a compartmentalized coculture model with mononuclear leucocytes and nonpathogenic bacteria. Scand I Immunol 60, 477-485.

24. Zeuthen LH, Fink LN \& Frøkiaer H (2008) Toll-like receptor 2 and nucleotide-binding oligomerization domain-2 play divergent roles in the recognition of gut-derived lactobacilli and bifidobacteria in dendritic cells. Immunology 124, 489-502.

25. Vossenkämper A, Marchès O, Fairclough PD, et al. (2010) Inhibition of NF- $\mathrm{BB}$ signaling in human dendritic cells by the enteropathogenic Escherichia coli effector protein NleE. J Immunol 185, 4118-4127.

26. Pagnini C, Saeed R, Bamias G, et al. (2010) Probiotics promote gut health through stimulation of epithelial innate immunity. Proc Natl Acad Sci US A 107, 454-459.

27. Plantinga TS, van Maren WW, van Bergenhenegouwen J, et al. (2011) Differential Toll-like receptor recognition and induction of cytokine profile by Bifidobacterium breve and Lactobacillus strains of probiotics. Clin Vaccine Immunol 18, 621-628.

28. Ma C, Muranyi $\mathrm{M}$, Chu $\mathrm{CH}$, et al. (2013) Involvement of DNA-PKcs in the IL-6 and IL-12 response to CpG-ODN is mediated by its interaction with TRAF6 in dendritic cells. PLOS ONE 8, e58072.

29. Kumar H, Kawai T \& Akira S (2011) Pathogen recognition by the innate immune system. Int Rev Immunol 30, 16-34.

30. Maldonado Galdeano C \& Perdigón G (2006) The probiotic bacterium Lactobacillus casei induces activation of the gut mucosal immune system through innate immunity. Clin Vaccine Immunol 13, 219-226.

31. Uematsu S, Fujimoto K, Jang MH, et al. (2008) Regulation of humoral and cellular gut immunity by lamina propria dendritic cells expressing Toll-like receptor 5. Nat Immunol 9, 769-776.

32. O'Callaghan J, Buttó LF, MacSharry J, et al. (2012) Influence of adhesion and bacteriocin production by Lactobacillus salivarius on the intestinal epithelial cell transcriptional response. Appl Environ Microbiol 78, 5196-5203.

33. Williams NT (2010) Probiotics. Am J Health Syst Pharm 67, $449-458$

34. Lebeer S, Vanderleyden J, De Keersmaecker SC, et al. (2008) Genes and molecules of Lactobacillus supporting probiotic action. Microbiol Mol Biol Rev 72, 728-764. 